\title{
Participation communautaire dans la référence évacuation des villages vers le CSCom central de Barouéli
}

\section{Community participation in the reference evacuation from villages to the central Community Health Center (CSCom) of Barouéli}

Ongoiba $\mathrm{S}^{1}$, Koné $\mathrm{K}^{1}$, Coulibaly $\mathrm{CA}^{1}$, Sangho $\mathrm{O}^{1,2}$, Telly $\mathrm{N}^{1}$, Sangho $\mathrm{F}^{1,3}$, Diarra $\mathrm{B}^{1}$, Sangho $\mathrm{H}^{1}$

\author{
1 Département d'Enseignement et de Recherche en Santé \\ Publique, Faculté de Médecine et d'Odontostomatologie, Boite \\ Postale 1805, Bamako, Mali. \\ ${ }^{2}$ DER des Sciences Biologiques et Médicales, Faculté de \\ Pharmacie, USTTB, Bamako, Mali \\ ${ }^{3}$ Faculté de Pharmacie, USTTB, Bamako, Mali
}

Auteur correspondant : Sadou Ongoiba, Master en Santé Publique, Option Santé Communautaire, 771490 33, ongoibasadou1@yahoo.fr

\section{Résumé}

La participation communautaire dans la référence/évacuation est définie comme l'ensemble des mécanismes et procédures utilisés pour mobiliser les ressources pour assurer le transport des urgences obstétricales et néonatales des villages vers le CSCom. Cette étude avait pour objectif d'explorer la participation communautaire dans la référence/évacuation des femmes et des enfants des villages au CSCom central de Barouéli. Matériel et Méthodes : II s'agissait d'une étude qualitative qui s'est déroulée du mois d'octobre 2017 au mois de février 2018 dans l'aire de santé de Barouéli central. Des entretiens individuels avec des chefs de ménages, des chefs de villages, les ATR, les présidentes des groupements de femme et le président ASACO et des groupes de discussion avec les responsables des caisses de santé villageoise et de solidarité ont été menés, associés à une analyse documentaire. Résultats: Notre étude montre que ce système de référence évacuation village-CSCom a été mis en place face à des difficultés d'accès aux soins de santé maternelle et néonatale, surtout les urgences obstétricales et néonatales. Ce système assure le transport des femmes en difficultés d'accouchement et des nouveaux nés des villages vers le Centre de Santé Communautaire de Barouéli central. Le système a permis d'évacuer 77 femmes sur 91 admirées (cahier de gestion du comité de référence/évacuation). Une absence totale de l'appui des collectivités au financement du système a été observée. Malgré ce manque d'appui, le système de référence évacuation des villages vers le CSCom a fonctionné de sa création jusqu'en fin 2017. Conclusion: Le système référence/évacuation villageCSCom assure aux femmes en difficultés d'accouchement d'accéder aux soins de santé maternelle et néonatale d'urgences et ne semble pas être affecté par l'absence de contribution financière de la collectivité. Mots clés : Référence/évacuation, Village-CSCom, urgence obstétricale et néonatale.

\begin{abstract}
Introduction: Community participation in referral I evacuation is defined as all mechanisms and procedures used to mobilize resources to transport obstetric and neonatal emergencies from the villages to the Community Health Center (CSCom). This study aimed to explore community participation in the referral I evacuation of women and children from villages to the central CSCom of Barouéli. Material and methods: This was a qualitative study which took place from October 2017 to February 2018 in the health area of central Barouéli. Individual interviews with heads of households, village chiefs, ATRs, the presidents of women's groups and the ASACO president and discussion groups with the heads of village health and solidarity funds were conducted, associated with a documentary analysis. Results: Our study shows that this village evacuationCSCom referral system was set up according to difficulties in accessing maternal and newborn health care, especially obstetric and neonatal emergencies. This system provides transport for women experiencing childbirth and newborns from the villages to the central Barouéli Community Health Center. The system made it possible to evacuate 77 women out of 91 admired (management book reference evacuation committee). A total lack of support from communities for funding the system was observed. Despite this lack of support, the reference system for evacuating villages to the CSCom worked from its creation until the end of 2017. Conclusion: The village-CSCom referral / evacuation system provides women with childbirth difficulties with access to emergency maternal and newborn health care and does not seem to be affected by the lack of financial contribution from the community.
\end{abstract}

Key words: Referral / evacuation, Village-CSCom, obstetric and neonatal emergency.

\section{Introduction}

Au Mali, selon l'Enquête Démographique et de Santé 2012-2013 (EDSM V) l'indice de mortalité maternelle est de 368 décès pour 100000 naissances vivantes, le taux de mortalité néonatale était de 35 pour 1000 au cours de la période de sept ans précédant l'enquête (2007-2013) [1]. Les taux de mortalité infantile et infanto juvénile sont respectivement de $56 \%$ et $95 \%$; ces taux élevés restent élevés comparativement au Sénégal, qui sont de $47 \%$ et $65 \%$ selon EDS Sénégal 2012-2013 [2]. L'EDSM V montre qu'au Mali trois femmes sur quatre (74\%) ont reçu des soins prénataux, dispensés par un prestataire qualifié dont sage-femme ou infirmière (44\%), matrone 
(25\%), médecin (4\%) et autre personnel formé (2\%) [1]. Malgré l'augmentation et l'amélioration des services offerts, l'accès des populations les plus pauvres et les plus vulnérables à ces services reste insuffisant surtout en milieu rural [1]. Près de six femmes sur dix (59\%) ont déclaré avoir des obstacles importants pour recevoir un traitement ou un avis médical [1]. La disponibilité de l'argent était le premier obstacle mentionné par $52 \%$ de ces femmes [1]. Ce taux passe de $41 \%$ en milieu urbain à $55 \%$ en milieu rural [1]. A cela s'ajoute la distance comme deuxième obstacle pour $36 \%$ des femmes interrogées [1]. Ce taux est quasiment doublé des milieux urbains (21\%) aux ruraux (40\%) [1]. Au-delà de ces deux obstacles, $29 \%$ des femmes affirment le besoin et le manque de permission d'un membre de la famille afin de pouvoir accéder aux soins de santé [1].

En 2015 le CSCom central de Barouéli a initié la mise en place d'un mécanisme sollicitant la participation communautaire dans la prise en charge des urgences obstétricales et néonatales. Pour faciliter l'accès aux soins obstétricaux et néonataux des villages vers le CSCom, les communautés se sont organisées en association afin de mettre en place des caisses de solidarité dénommées « Caisses Villageoises de Solidarité Santé (CVSS) ». Ces caisses ont été mises en place à l'image des expériences réussies dans certaines localités des régions de Kayes, Ségou et Koulikoro [2].

\section{Matériel et méthodes \\ Zones d'étude}

L'aire de santé de Barouéli central est située dans le district sanitaire de Barouéli, elle couvre 20 villages pour une population cible 35775 habitants (DNSI). Les femmes en âge de procréer représentent $5 \%$ de la population totale, soit 1789 femmes. L'aire de santé de Barouéli central est limitée au Nord par l'aire de santé de Boidié et de Dotembougou, à l'Est par l'aire de santé de Tigui, au Sud par l'aire de santé Konobougou, à l'Ouest par l'aire de santé de Niazana et N'djilla.

\section{Type d'étude et échantillon}

II s'agissait d'une étude transversale descriptive portant sur la participation communautaire dans la référence/évacuation des femmes et des enfants des villages vers le CSCom central de Barouéli. L'échantillon était composé de dix-huit chefs de ménages, trois chefs de villages, trois accoucheuses traditionnelles recyclées (ATR), trois présidentes de groupements de femmes, un président ASACO et neufs membres du comité de santé villageoise ( 3 pour chacun des 3 villages).

\section{Collecte des données}

Les données ont été collectées à travers les entretiens individuels et les groupes de discussion. L'outil utilisé a été le guide d'entretien en français mais traduit en bambara. A chaque catégorie de cible a été adressé un guide d'entretien. Ainsi l'entretien individuel a concerné les chefs de ménages et de villages, les présidents d'ASACO et de groupements de femmes et les ATR. Les groupes de discussions ont été animés avec les membres du comité de santé villageoise. Nous avons exploré le cahier de la référence/évacuation des villages au CSCom.

Les données recueilles ont fais l'objet d'un traitement manuel et classées par catégories. Elles ont été saisies et analysées avec le logiciel d'analyse qualitative Nvivo.

\section{Considérations éthiques}

Des correspondances ont été adressées au Médecin chef du district de Barouéli, au président de l'ASACO en vue d'obtenir leurs approbations pour la mise en œuvre de l'étude. Les consentements verbaux des participants étaient demandés et ils ont été rassurés de la confidentialité des informations recueillies. Pour cela un numéro d'identification a été utilisé pour masquer le nom et le prénom des participants.

\section{Résultats}

Nous avons enquêté au total 38 personnes dont 28 pour les entretiens individuels et 9 pour le groupe de discussions. II s'agissait de 18 chefs de ménages, 3 chefs de villages, 3 ATR, 3 présidentes de groupements de femmes, un président ASACO et 9 personnes pour les trois groupes de discussion (soit 3 personnes par groupe). Les caractéristiques sociodémographiques des 28 personnes sont décrites dans le tableau I. La tranche d'âge 46 - 55 ans des participants était majoritaire avec $64,29 \%$. La moyenne d'âge était de 51 ans, avec une étendu de 37 ans à 65 ans. Les hommes étaient majoritaires avec $78,57 \%$. La majorité des participants $(89,29 \%)$ était alphabétisés en langue locale. La plupart $(78,6 \%)$ était des cultivateurs

Selon les personnes interrogées, la mise en place du système de référence évacuation village-CSCom a fait suite à des problèmes identifiés dans l'aire de santé de Barouéli central. Les problèmes énumérés ont été une faible fréquentation du CSCom, la distance éloignée entre certains villages et le CSCom, l'absence de système organisé pour le transport des urgences obstétricales village-CSCom, l'incapacité pour un ménage seul à faire face aux besoins de déplacement et aux coûts en cas de complications obstétricales, la fréquence des complications survenues pendant le transport par charrettes tractées par des ânes et des cas de décès maternels dû à des moyens de transport inadéquats.

Ces difficultés énumérées ont été confirmées par les entretiens organisés avec les leaders communautaires, les membres de l'ASACO. Ils affirmèrent que c'est ce qui les a permis l'organisation d'assemblées générales d'information de l'ensemble de la population pour la recherche de solutions. A cet effet, pour assurer la référence évacuation des femmes en difficultés d'accouchement des villages vers le CSCom, les communautés se seraient organisées autour des caisses villageoises de santé et de solidarité.

De l'analyse des discours, il ressort qu'il existait deux organes qui permettaient le fonctionnement des caisses de santé villageoise dans l'aire de santé de Barouéli central: l'assemble générale et le comité de gestion. 
L'assemblée générale est l'instance suprême de prise de décision, elle est annuelle et désignes les membres du comité. Les comités étaient composés de 4 membres et présidés par un président issu de la communauté qui gérait les caisses villageoises de santé et de solidarités. Les autres 3 membres étaient un trésorier, un informateur et un administrateur (en charge aussi de gestion des courriers et des comptes rendus de réunions. Le Comité de gestion, disaient-ils, était chargé de la bonne marche du bureau, et assurait les activités de la référence/évacuation village-CSCom. Une difficulté évoquée était que les bureaux n'avaient jamais reçu de missions de supervision ni de la part de l'ASACO ni d'autres partenaires. Ce qui ne permettait pas un fonctionnement efficient du système et partant une gestion transparente de la caisse. Le président de la caisse villageoise de santé et de solidarité est le président du comité de gestion de la référence/évacuation village-CSCom. Ils ont expliqué que ce choix s'expliquait par la confiance, mais aussi par son capacité de mobiliser la communauté pour solutionner les éventuels problèmes de non payement des cotisations par les ménages.

Chaque année un compte rendu des activités serait fait à l'assemblée générale. Des rencontres mensuelles et trimestrielles seraient organisées, avec certains leaders communautaires (chef de village, ATR, relais communautaires), pour parler des activités menées durant les mois passés et l'état des caisses. Les membres du bureau interrogés affirment avoir été le plus souvent désignés sur la base de la confiance de la population et de leur disponibilité. Malgré les difficultés, toutes les caisses fonctionneraient à la satisfaction des membres du bureau rencontrés mais aussi de celle de la communauté (les bénéficiaires, les chefs des ménages). Les membres des comités de gestion des caisses étaient tous issus de la communauté. Les membres de l'ASACO ne venaient qu'en appuis et conseils. Les leaders communautaires étaient impliqués dans la gestion et les activités des caisses.

Pendant les entretiens individuels, les participants ont dans la très grande majorité répondu que les obstacles liés à la pérennité des caisses étaient entre autres la non participation de la collectivité et de l'état au financement du système de référence/évacuation village-CSCom, le refus de certains ménages d'adhérer au système, le non payement à temps des cotisations par certains ménages et la mauvaise gestion.

Les effets de la mise en œuvre de ce système de transport entre le village et le CSCom selon les participants étaient nombreux et divers. Tous les participants ont déclaré que le système a amélioré l'accessibilité géographique aux soins obstétricaux d'urgences, a réduit le temps de voyage, a contribué à l'amélioration de l'utilisation des services de santé maternelle et néonatale, a réduit le coût de l'évacuation et de la prise en charge des soins, a réduit la survenue des complications obstétricales. II y a eu une réduction des décès maternels et néonatals et a permis à toutes les couches socioéconomiques d'accéder aux soins de santé maternelle. D'autres ont signalé que grâce au système toutes les femmes dans le besoin avaient pu être évacuées à temps.

\section{Discussions}

Les limites de cette étude étaient entre autres le choix des participants de façon raisonnée avec ses conséquences sur l'impossibilité de généralisation de nos résultats, l'absence de support de gestion de la référence évacuation dans certains cas (ATR et certains comités de gestion de la caisse) influent sur le biais d'information, le biais de mémoire engendré par certains enquêtés ne se souvenaient plus des informations telles que la contribution faite par leurs ménages et comment la communauté participait à la gestion de la caisse de santé villageoise.

La mise en place des caisses villageoises de santé et de solidarités pour la référence/évacuation des femmes.

Les caisses de santé villageoise et solidarité dans l'aire de santé du CSCom central de Barouéli ont été mises en place pour faire face à des difficultés d'accès financier, la distance éloignée existant entre le village et le CSCom, l'absence du système organisé de la référence/évacuation village-CSCom, le manque de moyen financier d'un ménage seul à faire face aux besoins de déplacement et aux coûts en cas de complications obstétricales, la fréquence des complications survenues pendant le transport par charrettes à dos d'ânes et des cas de décès maternels dû à des moyens de transport inadéquats aux services de santé maternelle surtout les urgences obstétricales. Julie Fournier en 2011 avait trouvé qu'à Bafoulabé et Kita les difficultés d'accès financier et géographique étaient à l'origine de la création des caisses de santé villageoise et de solidarité de Dramétou, Ségala dans le cercle de Bafoulabé et Flabougou, Bangassi dans le cercle de Kita [3].

Nos résultats montrent que les caisses villageoises de santé et de solidarité dans l'aire de santé de Barouéli étaient des initiatives de la communauté. Depuis leur création en 2015, elles continuaient d'assurer les prestations jusqu'à notre enquête sur le terrain. Nous espérons que ça va continuer longtemps durant. En effet, ce cas du CSCom central de Barouéli semble prouver que les initiatives communautaires permettent d'assurer un certain degré de pérennité avec la participation communautaire.

Les groupements d'entraide comme les caisses villageoises de santé et de solidarité améliorent l'accès aux services de santé maternelle et obstétricale d'urgence. En Inde, Somen Saha et coll. ont montré que la présence d'un groupe d'entraide en zone rurale est associée à une amélioration du comportement sanitaire de la population [4] 
Nos résultats ont prouvé que les caisses assuraient le transport des urgences obstétricales et néonatales du village vers les CSCom. Les mêmes prestations étaient retrouvées dans l'étude de Julie en 2011 à Dramétou et Ségala [3].

Les moyens utilisés pour le transport des urgences obstétricales et néonatales par nos sites étaient la moto ambulance, les personnes interrogées trouvent que ce moyen est confortable, réduit le temps de transport et est moins cher comparée aux autres moyens, Dramétou et Ségala ne possédaient pas de moto ambulance, ils utilisaient un véhicule ambulance [3]. L'importance de la moto ambulance pour le transport des urgences obstétricales est retrouvée dans plusieurs études; I'UNICEF l'a démontré au Soudan du sud en 2013, cela est témoigné par les propos d'une mère de trois enfants bénéficiaire qui confirme "La moto ambulance a sauvé ma vie ", " je ne serais pas maintenant ici si je n'avais pas été transporté à l'hôpital par la moto ambulance» [5]. En 2008 au Malawi, Hofman JJ et coll. ont montré que dans les pays pauvres en ressources limitées, les motos ambulances dans les centres de santé ruraux sont un moyen de référence utile pour les soins obstétricaux d'urgence et une option relativement peu coûteuse pour le secteur de la santé [5].

Dans notre étude les résultats montrent que le premier recours des femmes enceintes dans les villages est l'accoucheuse traditionnelle recyclée et dans 1 cas sur 6 le premier recours est le CSCom. Cela peut s'expliquer par la proximité et l'accès facile des ATR par les parturientes au sein des villages.

Dans notre étude dix-sept femmes ont bénéficié du système de référence évacuation village-CSCom dans les dix-huit ménages enquêtés.

\section{Les obstacles liés à la pérennité des caisses}

Pendant les entretiens individuels, les participants ont dans la très grande majorité répondu que les obstacles liés à la pérennité des caisses sont entre autres la non participation de la collectivité et de l'état au financement du système de référence/évacuation village-CSCom, le refus de certains ménages d'adhérer au système, le non payement à temps des cotisations par certains ménages et la mauvaise gestion des caisses. L'étude menée par Fantamady CAMARA en 2017 montre que la mauvaise gestion, le non payement des cotisations et le départ de l'ONG ont entrainé l'arrêt de la caisse à Guémou Malinké [6].

Le niveau de satisfaction de la communauté sur la mise en œuvre du système

Les effets de la mise en œuvre de ce système de transport entre le village et le CSCom selon les participants sont nombreux et divers. Tous les participants ont déclaré que: le système a amélioré l'accessibilité géographique aux soins obstétricaux d'urgences, a réduit le temps de voyage, a contribué à l'amélioration de l'utilisation des services de santé maternelle et néonatale, a réduit le coût de l'évacuation et de la prise en charge des soins, a réduit la survenue des complications obstétricales. II y a eu une réduction des décès maternels et néonatals et a permis à toutes les couches socioéconomiques d'accéder aux soins de santé maternelle. D'autres ont signalé que grâce au système toutes les femmes dans le besoin ont pu être évacuées à temps et a facilité le transport et sauvé beaucoup de vie. L'étude menée par Fantamady CAMARA en 2017 a montré aussi que les caisses à Fassoudébé et à Guémou Malinké ont améliorées l'accessibilité financière et géographique des populations aux soins de santé. Elles ont aussi renforcé les liens d'entraide et de solidarité aussi bien chez les riches que chez les pauvres, contribués à une réduction du temps de transport et tout le monde a accès aux soins du CSCom grâce à ces caisses. [6].

\section{Les difficultés rencontrées dans la mise en place du système}

Pendant les entretiens individuels, tous les participants (28 personnes/28) ont répondu que la mise en place du système n'avait pas rencontrée de difficulté majeure en dehors de l'hésitation de certains ménages d'adhérer par manque de confiance aux membres de I'ASACO et du refus de la mairie de participer au financement du système. La mise en place du système a été facilitée par l'adhésion des chefs de village et aussi par le fait que les réunions sont effectuées chez le chef de village. Dans l'étude menée par Fantamady CAMARA en 2017 montre que la mauvaise définition des ménages, la non adhésion de certains ménages au système, une méfiance au vu des expériences antérieures non réussies, et l'incompréhension liée probablement à l'ignorance étaient les difficultés rencontrées dans la mise en place des caisses à Fassoudébé et à Guémou Malinké.

Prise de décision dans la famille pour la référence évacuation

Dans le cadre de la référence évacuation la prise de décision au niveau familiale est un aspect très important dans le processus. Les membres de la famille ont des responsabilités selon la configuration la familiale structurée de façon hiérarchique. La responsabilité de décision s'impose donc à la question de s'avoir qui doit décider du transfert de la femme en cas de difficulté d'accouchement au CSCom. L'ensemble des personnes enquêtées se disent le chef de famille étant le premier responsable est la personne habilitée à prendre la dernière décision de référer la femme au centre de santé ou en absence du chef de famille c'est la belle-mère ou la vieille mère qui décide de l'évacuation de la femme vers le centre de santé. Djibril Maïga en 2016 était parvenu aux mêmes résultats à Sanando et à Moabougou [7]. Dans notre étude les défis rencontrés étaient la non adhésion de certains ménages et le non payement des cotisations. Les raisons évoquées pour la plus part étaient le manque de confiance aux gestionnaires de la caisse et pour certains c'est la mauvaise volonté. Fantamady CAMARA en 2017 avait trouvé les mêmes défis à Guémou Malinké [6]. 


\section{Conclusion}

Le système de référence évacuation village-CSCom, depuis sa création en 2015 jusqu'en fin 2017, a mené des prestations de transport des urgences obstétricales et néonatales de façon régulière. Le système souffre d'une absence totale d'appui des collectivités en termes de financement. Malgré ce manque d'appui, toutes les femmes dans le besoin ont puis être évacuées à temps.

\section{Références bibliographiques}

1. Cellule de Planification et de Statistique (CPS/SSDSPF), Institut National de la Statistique (INSTAT/MPATP), INFO-STAT et ICF International, 2014. Enquête Démographique et de Santé au Mali 2012-2013. Rockville, Maryland, USA : CPS, INSTAT, INFO-STAT et ICF International.

2. Agence Nationale de la Statistique et de la Démographie (ANSD) [Sénégal], et ICF International. 2012. Enquête Démographique et de Santé Continue (EDS-Continue 2012-2013). Calverton, Maryland, USA: ANSD et ICF International.

3. Julie Fournier, Aliou Coulibaly, Caroline Tourigny, Soumaïla Diakité, Pierre Fournier. Une analyse de l'implantation des Caisses villageoises de solidarité-santé dans la région de Kayes, au Mali. 2013.

4. Saha S, Kermode M, Annear PL. Effect of combining a health program with a microfinance-based self-help group on health behaviours and outcomes. Public Health. nov 2015; 129(11):1510-8.

4. UNICEF, United Kingdom. Motorcycle Ambulances for Pregnant Women in South Sudan; Final report prepared for the Guernsey Overseas Aid Commission [Internet]. 2013 [cité 8 févr 2017]. Disponible sur: https://www.gov.gg/CHttpHandler.ashx?id=82788\&p=0.

5. Hofman JJ, Dzimadzi C, Lungu K, Ratsma EY, Hussein J. Motorcycle ambulances for referral of obstetric emergencies in rural Malawi: do they reduce delay and what do they cost? Int J Gynaecol Obstet Off Organ Int Fed Gynaecol Obstet. août 2008;102(2):191-7. 6. CAMARA F, 2017, Caisses villageoises de Solidarité Santé (CVSS) dans l'amélioration de l'état de santé des populations rurales de Diéma et de Nioro (Mali) mémoire pour un Master en santé publique, DRESP FMOS, Mali. 7. MAIGA D, 2016, Rôle des motos ambulances dans le cadre du transport des cas d'urgences obstétricales et néonatales des villages aux CSComs dans le district sanitaire de Barouéli (Mali) mémoire de fin d'étude cycle supérieur de développement Social, INFTS, Mali. 
Tableau I : Répartition des participants à l'enquête selon les caractéristiques sociodémographiques.

\begin{tabular}{|c|c|c|c|c|c|c|}
\hline caractéristiques & $\begin{array}{l}\text { Chefs de } \\
\text { ménages } \\
n=18\end{array}$ & $\begin{array}{l}\text { Présidente } \\
\text { groupement } \\
\text { de femme } \\
n=3\end{array}$ & $\begin{array}{l}\text { Chefs de } \\
\text { villages } \\
n=3\end{array}$ & $\begin{array}{l}\text { Président } \\
\text { ASACO } \\
n=1\end{array}$ & ATR & Total $n=28$ \\
\hline \multicolumn{7}{|l|}{ Tranche d'âge } \\
\hline $36-45$ & 2 & 1 & 0 & 0 & 0 & $3(10,71)$ \\
\hline $46-55$ & 11 & 2 & 2 & 0 & 3 & $18(64,29)$ \\
\hline $56-65$ & 5 & 0 & 1 & 1 & 0 & $7(25,00)$ \\
\hline \multicolumn{7}{|l|}{ Sexe } \\
\hline Masculin & 18 & 0 & 3 & 1 & 0 & $22(78,57)$ \\
\hline Féminin & 0 & 3 & 0 & 0 & 3 & $6(21,43)$ \\
\hline \multicolumn{7}{|c|}{ Niveau d'instruction } \\
\hline Primaire & 0 & 0 & 1 & 0 & 1 & $2(7,14)$ \\
\hline Secondaire & 0 & 0 & 1 & 0 & 0 & $1(3,57)$ \\
\hline Supérieur & 0 & 0 & 0 & 0 & 0 & $0(0,00)$ \\
\hline Alphabétisé & 18 & 3 & 1 & 1 & 2 & $25(89,29)$ \\
\hline sans instruction & 0 & 0 & 0 & 0 & 0 & $0(0,00)$ \\
\hline \multicolumn{7}{|l|}{ Activité menée } \\
\hline Cultivateur & 18 & 0 & 3 & 1 & 0 & $22(78,60)$ \\
\hline ATR & 0 & 0 & 0 & 0 & 3 & $3(10,70)$ \\
\hline Ménagère & 0 & 3 & 0 & 0 & 0 & $3(10,70)$ \\
\hline
\end{tabular}

